\title{
Cardiologia Baseada em Evidências I. Princípios, Racionalidade e Aplicações de uma Nova Prática Cardiológica e Análise Crítica da Literatura
}

\begin{abstract}
Álvaro Avezum
São Paulo, SP

\section{"Transferindo as Evidências da Pesquisa Clínica para a Prática Cardiológica"}

Recentemente, estamos vivenciando uma transição que já se encontra conosco, apenas não estando homogeneamente distribuída. Esta mudança é a do modelo da decisão clínica segundo opiniões isoladamente para o modelo da decisão, baseada em evidências. Isto conduz a uma avaliação crítica sistemática das informações disponíveis para a pratica da tomada de decisão clínica. A prática cardiológica requererá novas habilidades para praticar a decisão baseada em evidências, estruturando o que denominamos cardiologia baseada em evidências (CBE). De agora em diante devemos ter habilidade em definir critérios,

permitindo que princípios epidemiológicos (aliados a princípios de bioestatística) sejam aplicados às concepções, julgamentos, intuições, conhecimento, lógica e experiência prévia. Estes princípios epidemiológicos uma vez aplicados à cardiologia, permitem incrementos substanciais à eficácia, efetividade e eficiência das terapêuticas, como à acurácia diagnóstica. Como em medicina, e particularmente em cardiologia, nossa obrigação ética e moral é proporcionar mais benefícios do que riscos aos nossos pacientes, conseqüentemente, temos que interpretar crítica e adequadamente as informações, que são veiculadas através de publicações e apresentações, para podermos estar certos de que a nossa estratégia no manuseio do paciente (tomada de decisão) encontra-se correta.
\end{abstract} como eficácia, efetividade e eficiência, em avaliar a qualidade da evidência disponível e em incorporar e praticar os achados sólidos provenientes da pesquisa clínica. A CBEé a abordagem científica no manuseio clínico do paciente. A ciência de maior relevância na prática da medicina e, conseqüentemente, da cardiologia, é a epidemiologia clínica. A CBE, através da epidemiologia clínica, diferentemente da epidemiologia clássica, refere-se à aplicação dos princípios epidemiológicos no manuseio dos pacientes, detectando e diferenciando, assim, resultados que são clinicamente úteis e verdadeiros, daqueles que não o são ${ }^{1}$. A CBE oferece aos cardiologistas e clínicos, que praticam cardiologia, critérios específicos e objetivos a serem aplicados na avaliação da validade e utilidade clínica das informações obtidas, referentes à terapia, diagnóstico, prognóstico, metanálises, avaliações econômicas e avaliações de qualidade de vida. Virtualmente, a imensa maioria daqueles que pratica cardiologia (clínica e cirúrgica) obteve seu treinamento na prática clínica e cirúrgica e acredita estar praticando a arte da medicina derivada de concepções, julgamentos e intuições, como também acredita estar praticando a parte científica da medicina derivada do conhecimento, lógica e experiência prévia. Entretanto, recentemente temos observado que a ciência é a arte da cardiologia,

Instituto Dante Pazzanese de Cardiologia - São Paulo

Correspondência: Álvaro Avezum - Instituto Dante Pazzanese de Cardiologia Av. Dr. Dante Pazzanese, 500 - 12 $2^{\circ}$ - 04012-180 - São Paulo, SP

Recebido para publicação em 5/3/98

Aceito em 16/4/98

\section{Conceito}

Medicina baseada em evidências (MBE), cuja origem filosófica remonta na França, ao século 19, é a utilização conscienciosa, explícita e judiciosa da melhor evidência visando a tomada de decisão para o tratamento individual dos pacientes ${ }^{2}$. O termo MBE foi cunhado e primeiramente utilizado na Escola de Medicina da Universidade McMaster, Canadá, na década de 80 para denominar uma estratégia de aprendizado clínico ${ }^{3}$, envolvendo as seguintes etapas: 1) formular questões; 2) localizar as informações disponíveis na literatura; 3 ) avaliar criticamente as informações relevantes e 4) utilizar as informações avaliadas criticamente para a decisão clínica. Este processo tem como bases fundamentais os seguintes itens: 1 ) as decisões clínicas devem ser baseadas na melhor evidência científica disponível; 2) o problema clínico - ao invés de hábitos e protocolos - deve determinar o tipo de evidência a ser pesquisada; 3) a identificação da melhor evidência significa usar princípios epidemiológicos e bioestatísticos; 4) conclusões derivadas de evidências identificadas e avaliadas criticamente são úteis somente se influenciarem o manuseio de pacientes ou decisões sobre políticas de saúde e 5) o desempenho de quem prática MBE deve ser satisfatoriamente avaliado ${ }^{4-5}$. O termo CBE, originalmente introduzido no Brasil por Avezum ${ }^{6}$, envolve estas mesmas etapas aplicadas à cardiologia. A prática da CBE significa a integração da experiência clínica individual à melhor evidência externa disponível, proveniente da pesquisa clíni- 
ca. Devemos entender melhor evidência disponível por dados clinicamente relevantes da pesquisa. Com o exposto acima, depreende-se que bons cardiologistas utilizam experiência clínica individual e a melhor evidência científica disponível na prática cardiológica diária. A evidência clínica não deve tiranizar a experiência clínica, entretanto, sem evidências clínicas sólidas e robustas, a prática clínica torna-se desatualizada em detrimento do paciente. $\mathrm{O}$ questionamento fundamental é: qual a maneira correta de fazer as coisas corretamente?

O que a CBE não é? A CBE não é impossível de praticar, como também não é verdade que todos a estejam praticando. CBE não é um livro de receitas de medicina, pois requer uma abordagem que integre evidência externa, experiência clínica e, muitas vezes, preferências do paciente. A evidência clínica externa pode informar, mas nunca ocupar o lugar da experiência clínica individual. Esta integração permite a tomada de decisão clínica correta. Existe um certo receio de que a MBE seja utilizada por fontes pagadoras de serviços médicos, como também com fins gerenciais para cortar custos em saúde. Isto não apenas sugere um uso inadequado da MBE como também falta de entendimento das reais implicações financeiras da MBE. A prática da MBE, como também da CBE, identifica e aplica as intervenções mais eficientes, visando maximizar a qualidade e quantidade de vida para pacientes individuais. Esta prática pode tanto reduzir, como elevar os custos relacionados aos serviços de saúde. CBE não está restrita a estudos randomizados e metanálises. Por exemplo, para elucidar questões relativas a testes diagnósticos, necessitamos de estudos transversais adequados e para questões relativas ao prognóstico, necessitamos de estudos de coorte prospectivos ${ }^{5}$. O que pode e deve ser discutido é que para recomendarmos alguma intervenção terapêutica, necessitamos de estudos randomizados com poder estatístico adequado e bem conduzidos. Muitos estudos preliminares não deixam de ter seu valor, pois irão agregar ao corpo de evidências promissoras. Entretanto, o estudo definitivo deve ser realizado quando pretendemos recomendar determinada estratégia terapêutica. Quando não existirem estudos randomizados bem conduzidos, ou simplesmente, não existirem estudos randomizados, a prática da CBE dependerá das informações disponíveis, mesmo não sendo definitivas. O que existe de novo na CBE? 1) novos tipos de evidências têm sido geradas; 2) encontra-se nitidamente demonstrado que embora precisemos diariamente desta nova evidência, geralmente falhamos em obtê-la; 3 ) nosso conhecimento e desempenho clínico deteriora-se com o tempo; 4) tentativas de solucionar estes inconvenientes através de programas de educação médica continuada tradicional não melhora nosso desempenho clínico e 5) uma abordagem diferente no aprendizado clínico tem se mostrado eficiente para melhorar o desempenho clínico e atualizar o médico ${ }^{5,7}$. A CBE reforça, porém, nunca substitui julgamento clínico e experiência clínica.

\section{Racionalidade (por que a mudança de paradigma?)}

Nos últimos anos temos nos defrontado com um aumento no volume de informações obtidas através da literatura cardiológica, muitas das quais inválidas e, algumas vezes, irrelevantes para a prática clínica. A prática correta e desejável da cardiologia envolve a observação das seguintes premissas: efetuar o diagnóstico correto, selecionar o manuseio que proporcione mais benefícios do que riscos e manter-se atualizado no que concerne aos avanços e novidades em cardiologia. Os princípios epidemiológicos e bioestatísticos proporcionam o material necessário para os leitores aumentarem a acurácia e eficiência de seus esforços clínicos. Torna-se necessário e urgente o desenvolvimento de um filtro realmente científico para discernirmos a melhor forma de praticarmos a cardiologia. Para o desenvolvimento deste filtro necessitamos incorporar uma nova forma de aprender, pensar e agir dentro da cardiologia. Os princípios da epidemiologia clínica tornam-se, como referido por Sackett e col, "a ciência básica para a medicina clínica" 4 .O que todas as definições referentes à epidemiologia clínica têm em comum é um respeito pela melhor evidência possível sobre a eficácia e efetividade dos cuidados médicos e serviços de saúde num período de aumento de complexidade, no que se refere ao que podemos fazer por nossos pacientes e também um aumento no reconhecimento de que não devemos realizar determinadas condutas. Devido aos espaços conceituais, geralmente substanciais, entre a experiência estruturada da ciência básica e os problemas e discordância muitas vezes intermináveis da assistência clínica aos pacientes, surgiu a necessidade da incorporação da epidemiologia clínica com o objetivo de estruturar consistente e corretamente a prática clínica. Durante os últimos anos foi iniciado um processo de propagação e disseminação da necessidade de praticar uma MBE (em nosso caso particular, uma CBE) em diversos pontos do mundo em detrimento de uma medicina autoritária baseada apenas em opiniões de "peritos", que se baseiam em experiências pessoais (em geral por definição, experiência pessoal não utiliza grupos controles para analisar a eficácia de uma terapêutica). Apesar de profissionais que praticam recomendações baseadas em suas experiências, excluindo as evidências da literatura terem boa intenção, esta é uma prática autoritária, muitas vezes sem concordância entre os vários "peritos" e que podem nem sempre e nem todos estarem corretos. A falta de concordância entre as opiniões autoritárias estão relacionadas não apenas às diferentes condutas diagnósticas e terapêuticas, como também à validade das evidências sobre as quais suas recomendações repousam. O princípio "na minha experiência" utilizado de forma isolada, é um princípio que foi permitido no passado e que não deve ser mais praticado atualmente. Intuitivamente, o raciocínio embasado neste princípio faz sentido, entretanto este raciocínio não passa de uma impressão e deve ser checado através de níveis de evidência da literatura. Em muitas situações, devemos admitir a incerteza, encorajar o ceticismo e também ser- 
mos flexíveis para acomodarmos novas evidências que fatalmente chegam em nossas práticas e que podem contradizer o raciocínio e prática prévios. Evidências que não podem ser reduzidas à ciência de fato devem ser vistas com incerteza, suspeita e cautela ${ }^{6}$. Em resumo, intuições clínicas, experiências não sistemáticas e racionalidade fisiopatológica não são sinônimos de boa evidência para direcionar condutas em cardiologia. Existem critérios metodológicos para serem seguidos, indistintamente desde médicos residentes até chefes de departamento, e não a utilização de interpretações pessoais de resultados de estudos. Médicos pesquisadores necessitam entender as particularidades que envolvem o desenho de um protocolo, a obtenção dos dados e, posteriormente, a análise dos resultados. Contudo, os cardiologistas em geral, como os clínicos que praticam cardiologia, devem compreender os princípios básicos de pesquisa com o objetivo de interpretar o que foi encontrado no estudo publicado ou apresentado.

As barreiras, à introdução da CBE, podem ser divididas em: a) barreiras de atitude: hierarquia autoritária (pessoas diferentes emitindo opiniões diferentes sobre o mesmo paciente), antipatia profissional (resistência à mudanças), incerteza e preferências por métodos mais "fáceis" e b) barreiras práticas: método mais difícil, consumo de tempo, falta de disponibilidade de quem ensina e recursos de biblioteca (artigos e computadores) ${ }^{6}$.

\section{Como praticar CBE?}

Dentro da prática da CBE, cinco etapas são sugeridas: 1) converter a necessidade de informação em questões passíveis de esclarecimento; 2) procurar a melhor evidência disponível com a máxima eficiência; 3 ) avaliar criticamente as evidências encontradas em termos de validade (proximidade com a verdade) e utilidade (aplicabilidade clínica);4) aplicar os resultados da avaliação crítica na prática clínica e 5) avaliar nosso desempenho clínico contentemente. Os princípios fundamentais da CBE envolvem: 1) validade (podemos confiar na informação?); 2) importância (caso seja verdadeira, esta informação proporcionará uma diferença importante) e 3) aplicabilidade (como podemos usar esta informação?). Em resumo, julgamos que recomendações válidas são oriundas de evidências sólidas e robustas. O processo de decisão clínica compreende três componentes: experiência clínica, preferência do paciente e evidência da pesquisa. A CBE envolve a aplicação das evidências relevantes disponíveis provenientes da pesquisa clínica na prática cardiológica. Visando a introdução das evidências nas decisões clínicas, quatro etapas tornam-se cruciais: obtenção rápida das evidências, análise crítica, desenvolvimento de guias clínicos (critical pathways) e aplicação dos guias clínicos no local e tempo certos. Consegue-se assim, evidências rapidamente disponíveis para utilização em decisão clínica. Geralmente, centenas de estudos devem ser realizados para achar e constatar uma verdade clinicamente útil. As melhores informações veiculadas, através dos estudos clínicos, são publicadas em diversas revistas científicas; entretanto, o lei- tor muitas vezes nãoé adequadamente preparado do aparato necessário e essencial para criticar as informações e transformá-las em conhecimento real, ao invés de apenas consumir vorazmente as informações gerando um pseudoconhecimento. O número de estudos com resultados definitivos é muito pequeno e esses estudos encontram-se misturados com pequenos estudos preliminares, conflitantes, negativos ou que conduzem a falsos resultados (insuficiência metodológica parcial e total). A solução pode envolver o seguinte: guias clínicos para análise metodológica (não saia de casa sem ele), utilização da Medline e síntese e processamento de evidências clínicas de alta fidelidade (possível e fácil) e atualização apropriada em tempo real.

Anunciando a chegada da melhor evidência: quase todos os avanços no conhecimento médico são primeiramente apresentados na literatura médica. Conseqüentemente, a prática clínica deve ser orientada por análise crítica da literatura, devendo haver incorporação dos critérios objetivos e explícitos para selecionar informações científicas clinicamente relevantes e sólidas. No que diz respeito às fontes de evidências clínicas, torna-se importante para os leitores discernirem quais são os verdadeiros serviços baseados em evidências daqueles que não o são. Se a publicação não fornece regras explícitas para avaliação crítica, os leitores devem investir seu valioso tempo e recursos em outro lugar. O acesso eletrônico à literatura médica oferece baixa sensibilidade e especificidade para estudos relevantes, devendo o leitor desenvolver em si este filtro científico necessário, praticando deste modo uma CBE ao invés de uma prática clínica baseada apenas em opiniões alheias ${ }^{6}$.

A prática médica baseada em evidências estrutura-se em uma abordagem sistemática da literatura com questões claras e passíveis de resposta, avaliação crítica da validade e utilidade do que for encontrado, aplicação dos resultados aos pacientes e populações sob risco e avaliação do desempenho dos praticantes. O acesso ao conteúdo (itens que necessitam ser conhecidos) e ao processo (como aprender e aplicar os fatos), requer estilo e atitudes diferentes daquelas aprendidas na universidade e residência médica anteriores. As pessoas adquirem novos estilos e atitudes e tornam-se mais eficientes, ou seja, eficazes com a utilização adequada dos recursos disponíveis) ${ }^{7}$. Tendo estes princípios como base, podemos nos definir contra a seqüência da prática pseudocientífica: memorizar, regurgitar e graciosamente esquecer.

Durante as últimas décadas têm ocorrido uma expansão expressiva na pesquisa cardiovascular, resultando numa melhor compreensão da distribuição e determinantes da doença cardiovascular, como também os mecanismos biológicos básicos. Não podemos mais nos contentar em extrapolações baseadas na lógica derivada de dados experimentais, mas sim requerer provas sólidas e robustas de que um fator específico causa uma determinada doença ou de que um tratamento é efetivo através de estudos clínicos randomizados bem planejados e conduzidos. A cardiologia tem sido pioneira em inovações no planejamento e condução de grandes estudos, avaliando causalidade de doença 
(estudos caso-controle e de coorte), determinando prognóstico (banco de dados e registros), avaliando tratamentos (estudos randomizados amplos) e sintetizando informações através das revisões sistemáticas ou metanálises. Estes estudos têm aumentado em número e dimensão, suas qualidades têm se elevado e o impacto sobre a prática clínica é definitivamente crescente. Conseqüentemente, algumas questões são naturais: como o médico ocupado pode se atualizar diante desta explosão de conhecimentos? Como o médico julga quais estudos são válidos? Como o estudo encaixa no contexto de outras informações relevantes? Evidências podem ser derivadas de diferentes tipos de estudos, incluindo estudos epidemiológicos, estudos randomizados, registros, experimentos fisiopatológicos e animais. A evidência mais persuasiva deve ser não somente metodologicamente correta, mas deve também fornecer uma informação clinicamente relevante e coerente. Este conhecimento derivado desta matriz de informações deve ser transferido para a prática clínica à beira do leito ou em programas de prevenção na comunidade. A necessidade de treinar médicos de como transferir conhecimentos derivados do bons estudos (alta qualidade e impacto significante) à prática clínica diária requer arte e julgamento que são o coração da medicina baseada em evidências. Em certas ocasiões, a CBE deve ser aplicada às áreas onde as evidências ainda não são completas, onde podemos denominar CBE na zona indefinida. O conhecimento veiculado pela CBE deve conter informações clinicamente relevantes, de alta qualidade metodológica, informativa, rápida e sucinta ${ }^{8}$.

Quando procuramos a melhor evidência disponível para solucionarmos um problema clínico devemos tentar preencher 3 itens: a questão, nossa melhor resposta, nossa evidência inicial para resposta. Existe mais de uma via adequada de acesso à melhor evidência, entretanto existem várias vias inadequadas e incorretas. Torna-se necessário a disponibilidade e qualidade de recursos para obter as melhores evidências.

Quanto à Medline, devemos utilizar os seguintes termos para pesquisa: clinical trial para terapia, sensitivity para testes diagnósticos, risk para etiologia, cohort Studies para prognóstico, meta-analysis ou review para revisões sistemáticas ${ }^{9}$. Decididamente, hoje temos acesso à melhor evidência disponível sem precedentes.

\section{Como enunciar questões clínicas passíveis de respostas?}

Todas as vezes que atendemos um paciente, necessitamos de informações novas sobre algum elemento em relação ao diagnóstico, prognóstico e manuseio clínico. Algumas vezes a questão é auto-explicativa e a resposta é facilmente obtida. Outras vezes, necessitamos transformar o problema clínico em questão passível de ser esclarecida. Existem diferentes áreas para questões na prática clínica: 1) achados clínicos: como interpretá-los através da história clínica e exame físico; 2) etiologia: como identificar causas para doenças; 3) diagnóstico diferencial: quando considerar as possíveis causas de um problema clínico, como classificá-lo de acordo com probabilidade, seriedade e for- mas de tratamento; 4) testes diagnósticos: como selecionar e interpretar testes diagnósticos, visando confirmar ou excluir um diagnóstico, considerando sua precisão, acurácia, aceitação, custos e segurança; 5) prognóstico: como estimar a probabilidade do curso clínico do paciente e antecipar a probabilidade de complicação da doença; 6) terapia: como selecionar tratamentos que ofereçam mais benefícios do que prejuízos ao paciente, incluindo os custos; 7) prevenção: como reduzir a chance da doença através da identificação e modificação dos fatores de risco, e como diagnosticar a doença precocemente através de screening; 8) automelhoria: como manter-se atualizado, melhorar o estilo clínico e tornar a prática clínica mais eficiente ${ }^{5}$.

Em geral, as questões bem estruturadas apresentam os seguintes elementos: 1) o paciente ou problema endereçado; 2) a intervenção considerada; 3) a intervenção de comparação quando relevante e 4) o resultado clínico de interesse.

\section{Avaliação crítica das evidências disponíveis na literatura (como ler corretamente os artigos científicos sobre terapia)}

Uma questão freqüentemente enunciada por todos nós, quando pretendemos empregar a melhor conduta terapêutica para nossos pacientes é "como os resultados deste artigo se relacionam com o meu paciente?" ${ }^{10}$.Como podemos observar, existe uma estreita ligação da revista científica com a manuseio clínico do paciente. Chamamos esta conexão de epidemiologia clínica à beira do leito, ou seja, a aplicação dos resultados de estudos clínicos aos nossos pacientes individuais. Não basta apenas lermos os resultados e conclusões para conhecermos o assunto e conseqüentemente tratarmos os pacientes. Temos obrigatoriamente que analisar criticamente os resultados através de critérios simples e objetivos para sabermos sobre a validade dos mesmos. Na presença de dados confiáveis e válidos, não podemos ceder espaço para o subjetivismo e impressão pessoal para conduzirmos clinicamente nossos pacientes. Felizmente, estudos clínicos controlados e randomizados estão sendo realizados com mais freqüência e têm demonstrado de forma clara a eficácia de alguns tratamentos, como também a ausência de utilidade clínica ou mesmo o risco de tantos outros. Entretanto, a avaliação correta de uma terapia requer mais do que randomização. Discutiremos os critérios que devem ser seguidos para uma avaliação crítica de um artigo distinguindo com isto resultados úteis, conflitantes, confusos ou mesmo prejudiciais. De forma didática, estes critérios são divididos em três grupos: validade dos resultados (quadro I); quais são os resultados (quadro II) e como estes resultados podem ser incorporados ao manuseio de pacientes (quadro III).

Os resultados do estudo são válidos? Esta questão refere-se à validade ou acurácia dos resultados e considera se o efeito do tratamento relatado no artigo representa a verdadeira direção e magnitude do efeito do tratamento. Uma outra maneira de formular e compreender esta questão seria a seguinte: estes resultados representam uma estimativa sem 
Quadro I - Critérios para a avaliação crítica de um artigo sobre terapia ${ }^{6,11}$ Os resultados do estudo são válidos?

Critérios primários:

1. A designação dos pacientes para os tratamentos foi realmente randomizada?

2. Todos os pacientes admitidos no estudo foram adequadamente contados e atribuídos à conclusão?

3. O seguimento foi completo?

4. Os pacientes foram analisados nos grupos em que foram originalmente randomizados?

Critérios Secundários:

1. Os pacientes, médicos e pessoal envolvido no estudo apresentavamse sob a condição cega no que diz respeito ao tratamento?

2. Os grupos comparados eram similares no início do estudo?

3. Além da intervenção experimental do estudo, os grupos comparados foram tratados igualmente?

\begin{tabular}{|l|}
\hline $\begin{array}{c}\text { Quadro II - Critérios para a avaliação crítica de um artigo } \\
\text { sobre terapia }\end{array}$ \\
Quais foram os resultados? \\
\hline 1. Qual a dimensão do efeito do tratamento? \\
2. Qual a precisão da estimativa do efeito do tratamento? \\
3. As significâncias clínica e estatística foram consideradas?
\end{tabular}

\footnotetext{
Quadro III - Critérios para a avaliação crítica de um artigo sobre terapia ${ }^{6,12}$

Os resultados irão auxiliar-me no manuseio de meus pacientes?

1. Os resultados podem ser aplicados no manuseio de meus pacientes? (os pacientes do estudo eram similares aos meus pacientes e o manuseio utilizado pode ser também utilizado na minha prática clínica).

2. Todos os objetivos clinicamente relevantes foram considerados?

3. Os benefícios do tratamento superam os riscos potenciais e os custos?
}

erros sistemáticos do efeito do tratamento ou apresentam alguma influência de forma sistemática conduzindo a uma falsa conclusão?

Quais foram os resultados? Caso os resultados sejam válidos e o estudo provavelmente conduz a uma avaliação do efeito do tratamento sem erros sistemáticos, então os resultados têm valor para serem examinados. Esta segunda questão considera a dimensão e a precisão do efeito do tratamento. A melhor estimativa deste efeito será os achados do estudo por si e a precisão da estimativa será superior em estudos com maior número de pacientes.

Os resultados auxiliar-me-ão no manuseio de meus pacientes? Esta questão apresenta dois itens: a) os resultados são aplicáveis aos seus pacientes? Você deveria hesitar em instituir e incorporar o tratamento se seus pacientes não são similares àqueles do estudo, ou se os objetivos onde foi demonstrado benefício não forem suficientemente importantes para os seus pacientes; b) se os resultados são aplicáveis, qual é o impacto do tratamento? O impacto depende dos benefícios e riscos (efeitos adversos e tóxicos) do tratamento e das consequiências de deixar de tratar os pacientes. Portanto, mesmo um tratamento efetivo poderá não ser utilizado quando o prognóstico do paciente já é bom sem este tratamento, especialmente quando estiver associado a importantes efeitos adversos e alto custo.

\section{Os Resultados do Artigo são Válidos?}

\section{Critérios primários}

A designação dos pacientes para os tratamentos foi realmente randomizada? O processo de randomização permite estabelecer similaridade entre os dois grupos comparados (ex: tratamento A versus controle), no que diz respeito a fatores de risco conhecidos como desconhecidos. Diferentes resultados podem ser obtidos quando o tratamento em comparação é designado de forma randomizada ou quando esta designação é realizada de forma consciente pelo investigador. Determinados fatores, como a severidade da doença, presença de comorbidade e outros fatores prognósticos conhecidos e desconhecidos, podem alterar positiva ou negativamente o efeito de uma terapia em investigação clínica. Como estes fatores descritos podem influenciar a decisão médica, estudos de eficácia não-randomizados são inevitavelmente limitados, quanto a sua habilidade em distinguir se a terapia é útil ou não, como também se existem riscos com a utilização da mesma. A confirmação do exposto, encontra-se no fato de que designação de tratamentos, através de outras maneiras que não a randomização, tende a produzir efeitos maiores (e freqüentemente falso-positivos) de tratamento do que em estudos randomizados ${ }^{6}$. Desde que o tamanho da amostra seja suficiente, a randomização assegura que determinantes prognósticos conhecidos e desconhecidos sejam distribuídos igualmente entre os grupos tratamento e controle. O método de randomização é outro critério a ser avaliado, visto que métodos que utilizam códigos fornecidos por computador ou de maneira automatizada, através de ligação telefônica, onde os critérios de inclusão e exclusão são checados novamente antes de informar qual o tratamento designado, reduzindo as chances de saber qual o tratamento a ser designado antes de se realizar a randomização, são preferíveis àqueles que utilizam envelopes.

Todos os pacientes admitidos no estudo foram adequadamente contados e atribuídos à conclusão? Neste item temos dois componentes: a) O seguimento foi completo? Todo paciente que foi admitido no estudo deve ser analisado na sua conclusão final. Caso isto não seja feito ou um número substancial de pacientes seja relatado como "perda de seguimento", a validade do estudo está aberta para questionamentos. Quanto maior o número de pacientes com perda de seguimento, mais o estudo está sujeito a erros sistemáticos, porque os pacientes que estão perdidos freqüentemente têm prognósticos diferentes do que aqueles onde o seguimento foi adequadamente realizado. Em casos de perda de seguimento, duas possibilidades influenciando os resultados do estudo de forma diferente, podem ocorrer: 1) os pacientes não retornaram para o seguimento porque apresentaram resultados adversos, incluindo óbito; 2) os pacientes estão clinicamente bem e devido a isto não retornaram à visita clínica para serem avaliados. Os leitores podem decidir se a perda de seguimento foi excessiva assu- 
mindo em estudos com resultados positivos (o tratamento em investigação promove resultados benéficos), que todos os pacientes perdidos no grupo tratamento evoluíram mal (ex: apresentaram óbito) e todos aqueles perdidos no grupo controle evoluíram bem (ex: encontram-se vivos). Recalculando-se os resultados sob esta circunstância proposta, se as conclusões do estudo não forem modificadas, a perda de seguimento não foi excessiva. Entretanto, caso as conclusões se modifiquem, a perda de seguimento deve ser considerada excessiva e o poder de inferência, através da conclusão obtida, torna-se enfraquecido e os resultados não são confiáveis ${ }^{6,11}$. b) os pacientes foram analisados nos grupos em que foram originalmente randomizados? Na prática clínica, pacientes em estudos randomizados esquecem algumas vezes de utilizar a medicação ou mesmo recusam-se à esta utilização. À primeira vista, os leitores concordarão que pacientes que nunca utilizaram a medicação designada deveriam ser excluídos da análise de eficácia. Freqüentemente, as razões pelas quais os pacientes não utilizam a medicação estão relacionadas ao prognóstico. Alguns estudos clínicos randomizados demonstram que pacientes não aderentes ao tratamento apresentam pior prognóstico do que aqueles que o são, mesmo considerando-se todos os fatores prognósticos conhecidos e incluindo aqueles pacientes que utilizaram placebo ${ }^{6}$. A exclusão de pacientes não aderentes ao tratamento da análise destrói a comparação sem erros sistemáticos, proporcionada pela randomização. Este princípio de atribuir todos os pacientes aos grupos nos quais eles foram originalmente randomizados denomina-se análise de intençãode-tratar. Esta estratégia preserva o valor da randomização, permitindo a distribuição balanceada de fatores prognósticos nos grupos comparados e, conseqüentemente, o efeito observado será realmente devido ao tratamento designado.

\section{Critérios secundários}

Os pacientes, médicos e pessoal envolvido no estudo apresentavam-se sob a condição cega no que diz respeito ao tratamento? Pacientes que sabem que estão utilizando um novo tratamento são mais prováveis de terem uma opinião sobre sua eficácia, como também médicos ou pessoas envolvidas com as avaliações sobre a resposta ao tratamento em questão. Estas opiniões, se otimistas ou pessimistas, podem distorcer sistematicamente os aspectos e o relato dos objetivos do tratamento, reduzindo, conseqüentemente, a confiança nos resultados do estudo. Devido ao fato de pessoas envolvidas na avaliação dos objetivos do estudo não estarem sob a condição cega, podem ser fornecidas diferentes interpretações dos achados durante a realização dos exames em questão, distorcendo assim os resultados. A forma mais aconselhável para evitar este risco à validade é a realização de estudos duplo-cegos (paciente e médico e/ ou pessoal envolvido no estudo não são capazes de distinguir o tratamento ativo do placebo).

Os grupos comparados eram similares no início do estudo? Com o objetivo de se assegurarem quanto à valida- de do estudo, os leitores devem ser informados se os grupos tratamento e controle eram similares com relação aos fatores que podem influenciar e determinar os resultados clínicos de interesse. Estes dados são obtidos através da demonstração das características basais dos grupos tratamento e controle. Embora nunca saibamos se existe similaridade entre os grupos em relação a fatores prognósticos não conhecidos, podemos nos assegurar que os fatores prognósticos conhecidos estão adequadamente balanceados. Quando aumentamos o tamanho da amostra, os grupos tornam-se balanceados, tornando mais difícil o efeito do acaso (play of chance), sugerindo que um grupo tem aparentemente prognóstico melhor do que o outro. Em estudos adequadamente randomizados com tamanho de amostra suficiente, sabemos antecipadamente que qualquer diferença entre os grupos tratamento e controle ocorre por acaso, independentemente se houve ou não significância estatística. Entretanto, o ponto em questão é a magnitude desta diferença. Se for uma grande diferença, a validade do estudo torna-se comprometida. Quanto maior a relação entre fatores prognósticos e resultados e quanto menor o tamanho da amostra do estudo, mais as diferenças entre os grupos enfraquecerão a força de qualquer inferência sobre eficácia. Felizmente, quando os grupos não são similares quanto às características basais, algumas técnicas estatísticas permitem o ajuste dos resultados do estudo para as diferenças entre estas características ${ }^{6,11}$. Obviamente, devemos procurar similaridade entre características relevantes e quando tanto a análise ajustada quanto a não-ajustada para as características diferentes permitirem a mesma conclusão, admitiremos de forma justificada a validade dos resultados do estudo.

\footnotetext{
Além da intervenção experimental do estudo, os grupos comparados foram tratados igualmente? As formas de tratamento nos grupos tratamento e controle podem diferir entre si de várias maneiras e estas diferenças podem também distorcer os resultados do estudo. Intervenções outras além daquela envolvida no estudo são chamadas de co-intervenções e quando aplicadas diferentemente aos grupos tratamento e controle podem alterar os resultados. A co-intervenção é um problema sério principalmente quando a condição duplo-cega está ausente. Como exemplo, podemos ter um estudo avaliando pacientes pós-infarto do miocárdio com determinado tratamento e durante o seguimento um dos grupos (tratamento ou controle) receber procedimentos de revascularização miocárdica com mais freqüência. A co-intervenção neste caso são os procedimentos de revascularização miocárdica, os quais por terem sido mais utilizados em um grupo poderão influenciar os resultados, favorecendo um dos grupos e com isto salientar ou atenuar os efeitos do tratamento em estudo.

Os itens discutidos acima auxiliarão o leitor a determinar se os resultados do artigo são provavelmente válidos ou não. Se os resultados forem considerados válidos, então o leitor poderá prosseguir e considerar a magnitude do efeito do tratamento e sua aplicabilidade.
} 


\section{Quais foram os resultados?}

Qual a dimensão do efeito do tratamento? Existem várias maneiras de se expressar os efeitos de um tratamento: a) redução absoluta de risco (RAR): proporção de eventos no grupo controle menos a proporção de eventos no grupo tratamento $(\mathrm{ex}: 0,10-0,085=0,15)$; b) risco relativo (RR): proporção de eventos no grupo tratamento relativa à proporção de eventos no grupo controle (ex: 0,085/0,10= $0,85)$; c) redução relativa de risco (RRR): 1 menos proporção de eventos no grupo tratamento relativa à proporção de eventos no grupo controle vezes $100 \%$ [ 1 - $(0,085 / 0,10)$ $\mathrm{X} 100 \%=15 \%]$. Esta é a medida mais comumente utilizada, significando neste exemplo que o novo tratamento reduziu o risco de eventos em $15 \%$ em comparação com o grupo controle. $^{6,12}$.

Qual a precisão da estimativa do efeito do tratamento? A verdadeira redução de risco nunca pode ser conhecida. Tudo o que temos é uma estimativa fornecida por estudos clínicos controlados randomizados, e a melhor estimativa do verdadeiro efeito do tratamento é aquela observada nestes tipos de estudos. Temos então um ponto de estimativa devido ao fato da impossibilidade de sermos precisamente corretos. Conseguimos saber os limites (vizinhança) dentro dos quais o verdadeiro efeito provavelmente se encontra. Esta estratégia estatística denomina-se intervalos de confiança (ICs) ${ }^{13}$. Geralmente (embora arbitrariamente), utilizamos IC 95\%, o qual é interpretado como definindo a faixa que inclui a verdadeira RRR 95\% das vezes. Significa que a verdadeira RRR encontrar-se-á destes limites em 95\% das vezes. Quanto maior o tamanho da amostra do estudo, maior será o número de eventos e maior será nossa confiança de que a verdadeira RRR (ou outra medida de eficácia) estará próxima daquela que observamos. Tornase evidente que quanto maior o tamanho da amostra, mais estreito é o IC. A precisão de um resultado de estudo é fornecida pelo IC e não pelo valor de p, ou seja, mesmo com resultados estatisticamente significantes, podemos ter falta de precisão devido a amplos ICs e, conseqüentemente, a importância clínica do resultado estará sujeita a esta falta de precisão. Se, por exemplo, obtivermos uma RRR de $25 \%$ com IC $95 \%-8 \%$ a $-40 \%$, significa que se repetirmos o experimento 100 vezes, em 95 das vezes a RRR encontrar-se-á entre 8 e 40. Obviamente, uma RRR de $8 \%$ tem uma importância clínica diferente de uma de $25 \%$ ou mesmo de $40 \%$. Quando o artigo não fornece este importante elemento para avaliarmos a precisão dos resultados obtidos, devemos proceder da seguinte forma: 1) caso o valor de p seja igual a 0,05 , significa que o limite inferior do IC para a RRR é zero (não se pode excluir que o tratamento não tenha efeito). Conforme o valor de p diminui abaixo de 0,05, o limite inferior para a RRR eleva-se acima de zero; 2 ) quando o artigo fornece o erro padrão da RRR (ou do RR), o limite inferior e superior do IC 95\% para uma RRR são os pontos estimados mais e menos duas vezes este erro padrão; 3) calcular o $\mathrm{IC}^{6,12}$.
As significâncias clínica e estatística foram consideradas? Significância clínica neste contexto refere-se à importância da diferença nos resultados clínicos entre os grupos tratamento e controle, sendo geralmente descrita em termos de magnitude de um resultado. Em contraste, significância estatística mostra se as conclusões obtidas pelos autores apresentam probabilidade de serem verdadeiras, independentemente de serem clinicamente importantes ou não. Duas questões devem ser formuladas para se avaliar este item: 1) além de estatisticamente significante, a diferença encontrada também é clinicamente significante?; 2) se a diferença nãoé estatisticamente significante, o estudo apresentava um tamanho de amostra suficiente para demonstrar uma diferença clinicamente importante, caso esta tenha ocorrido?

Como exemplo, imaginamos que um autor conclui que o tratamento Aé melhor do que B, com um valor de $\mathrm{p}=0,01$. Em outras palavras, o valor de $\mathrm{p}$ nos diz o quão freqüente os resultados poderiam ter ocorrido por acaso, se a intervenção não fosse diferente do controle. Isto significa que o risco de ter concluído erroneamente que A é melhor do que B (quando na verdade não o é) é de apenas $1 \mathrm{em} 100$. Isto nos parece bom, entretanto a questão é: "isto é clinicamente importante também?". Significância clínica vai além da aritmética e é determinada por julgamento clínico. A medida utilizada para avaliar a significância clínica é o número necessário para tratar (NNT), o qual têm três propriedades para ilustrar sua utilidade clínica: a) enfatiza os esforços utilizados para atingir um alvo tangível de tratamento (auxilia a quantificare desmistificar a decisão de tratar alguns, mas não todos os pacientes); b) fornece a base para se expressar os custos do tratamento; c) fornece elementos úteis para comparar diferentes tratamentos para diferentes doenças ${ }^{6,12,14}$.

Após a determinação da magnitude e precisão do efeito do tratamento, a questão final refere-se a como aplicar os resultados do artigo aos pacientes e na prática clínica.

\section{Os resultados auxiliar-me-ão no manuseio de meus pacientes?}

Os resultados podem ser aplicados no manuseio de meus pacientes? Se os pacientes preencherem os critérios de inclusão e exclusão estabelecidos pelo estudo em questão, não há muita discussão quanto à aplicabilidade dos resultados. Entretanto, se este não for o caso, torna-se necessário um outro julgamento e uma outra análise. Ao invés de argumentarmos que nosso paciente é dois anos mais novo ou o nível de pressão arterial sistólica é $2 \mathrm{mmHg}$ diferente do que aquele estipulado pelo estudo, devemos perguntar se existe alguma razão que torna os resultados não aplicáveis aos nossos pacientes. Em geral, uma razão significativa não é encontrada, tornando assim os resultados possíveis de serem generalizados para nossos pacientes com razoável confiança. Cuidado especial deve ser tomado quando os resultados globais do estudo não demonstram diferenças significativas e através de análises de subgrupos realizadas posteriormente (sem hipótese pré-especificada) demonstram que determinados subgrupos de pacientes se benefi- 
ciam do tratamento. Resumidamente, para generalizarmos os achados de um subgrupo particular aos nossos pacientes, devemos seguir as seguintes etapas em relação ao efeito de tratamento: a) é amplo?; b) é improvável ter sido obtido por acaso?; c) a hipótese foi especificada antes do estudo ter sido iniciado?; d) os achados foram também encontrados em outros estudos (replicabilidade) $?^{6,12}$. Os leitores em geral devem ser céticos quanto à aplicação de resultados de análises de subgrupos para seus pacientes.

Todos os objetivos clinicamente relevantes foram considerados? Tratamentos são indicados quando fornecem importantes benefícios. Demonstração de que um vasodilatador melhora o débito cardíaco ou de que um antiarrítmico promove supressão de arritmia ventricular não necessariamente fornecem suficiente razão para a prescrição destes tratamentos. O que se torna necessário é a evidência de que estes tratamentos atuem positivamente em objetivos que são definitivamente importantes para o paciente, como por exemplo, redução de hospitalização, redução de mortalidade ou redução do risco de infarto do miocárdio. A escolha e a utilização de objetivos substitutos (surrogate endpoints) deve ser cuidadosa, devendo não apenas refletir o objetivo principal como assegurar que a interferência positiva de um tratamento sobre um objetivo substituto apresente igualmente interferência sobre o que se procura substituir (ex: mortalidade) ${ }^{6,12,15}$. Um exemplo do risco na escolha de objetivos secundários ocorreu no estudo CAST I e II ${ }^{16,17}$. Os antiarrítmicos (encainida, flecainidae moricizina) utilizados para tratamento de arritmias ventriculares complexas pós-infarto, causaram aumento nas taxas de mortalidade, a despeito de se mostrarem eficazes na supressão destas arritmias. Mesmo quando os autores relatam efeitos favoráveis do tratamento sobre determinados objetivos, os leitores devem se certificar de que não ocorre nenhum efeito deletério sobre outros objetivos.

Os benefícios do tratamento superam os riscos potenciais e os custos? Antes da decisão de iniciar determinado tratamento, deve-se considerar os riscos do paciente em apresentar o evento se for deixado sem tratamento. Quanto maior a possibilidade de um paciente apresentar um evento caso não tratado, mais provável torna-se o benefício do tratamento para este paciente e menos pacientes deverão ser tratados para prevenir um evento. O número de eventos adversos decorrentes do uso do tratamento novo em cada 1.000 pacientes tratados deve ser contabilizado na decisão final. A incorporação deste risco ao ser incorporada, permite uma análise global e verdadeira da eficácia (ex: a eficácia do ativador tecidual do plasminogênio em comparação com a estreptoquinase, deve ser vista como número de vidas salvas mais o número de sangramento intracraniano por cada 1.000 pacientes tratados). Análises econômicas, principalmente análise de custo-efetividade, a qual considera a eficácia do tratamento e os custos envolvidos na obtenção de sua eficácia são altamente desejáveis. O princípio "sempre os recursos serão escassos", utilizado inclusive em paí- ses desenvolvidos, deve ser acentuadamente observado em nosso meio, principalmente tendo em vista o panorama econômico de nosso país. Quando se assume que os recursos sempre serão escassos, a utilização eficiente dos tratamentos dependerá da designação destes recursos para determinados tratamentos e situações, os quais devem apresentar evidências sólidas de eficácia terapêutica.

Adicionalmente aos critérios acima podemos complementar nossa avaliação metodológica visando o conduta à beira do leito através das seguintes questões: 1) os resultados são convincentes em qual magnitude? Geralmente, um estudo é mais convincente se a diferença em um objetivo primário pré-definido atinge um nível extremo de significância estatística e a análise é baseada em todos pacientes randomizados (análise por intenção de tratar). Devemos lembrar que um estudo que atinge o nível nominal de significância de $\mathrm{p}<0,05$ apresenta apenas $50 \%$ de chance de ser replicado se o mesmo experimento for repetido. Se obtivermos $\mathrm{p}<0,01$, esta chance será de $70 \%$, enquanto que $\mathrm{p}<0,001$ indica uma chance de replicação de $90 \%$. Consequientemente, a decisão de tratamento requer valores mais extremos de p. Devemos ser cautelosos quando através de inúmeras análises retrospectivas e não pré-especificadas, o autor propõe que uma determinada significância estatística foi atingida. Este achado pode ser devido apenas ao efeito do acaso, merecendo replicação para ser considerado verdadeiro; 2) as inferências são baseadas em uma quantidade de dados suficientes para que os resultados sejam robustos? Existem dois tipos de erros comuns em um estudo clínico mesmo quando bem realizado. Quando existe uma diferença nítida e o estudo não demonstra esta diferença, denominamos resultado falso negativo (erro tipo II ou erro beta). Neste caso temos um estudo com baixo poder estatístico. Um item mais complexo é quando o tratamento realmente não é eficaz, porém os resultados são estatisticamente significantes, ou resultado falso positivo (erro tipo I ou erro alfa). O erro tipo I é incomum em estudos bem conduzidos, ocorrendo em chances substancialmente mais elevadas em estudos pequenos. Devemos ser céticos quando determinados autores clamam por benefícios substanciais, em geral não esperados e baseados em pequeno número de pacientes; 3) os resultados foram replicados ? O que cardiologistas e clínicos que praticam cardiologia querem saber é se os resultados demonstrados em determinados estudos podem ser replicados quando largamente utilizados na prática diária ${ }^{10}$. Naturalmente, temos uma confiança maior quando outros estudos chegam à conclusões similares (exemplo: terapia trombolítica, betabloqueadores, aspirina, inibidores da enzima conversora da angiotensina no tratamento do infarto agudo do miocárdio).

Em resumo, a aplicação dos resultados de estudos clínicos requer não somente conhecimento dos estudos, mas interpretação cuidadosa, visando examinar a consistência dos resultados. A avaliação crítica da literatura envolve ainda as seguintes áreas que serão discutidas nas publicações posteriores: prognóstico, testes diagnósticos, revisões sistemáticas (metanálises) e análises econômicas. 
Figura 1 - Questionário para avaliação crítica de um artigo sobre terapia em cardiologia ${ }^{6,11,12}$

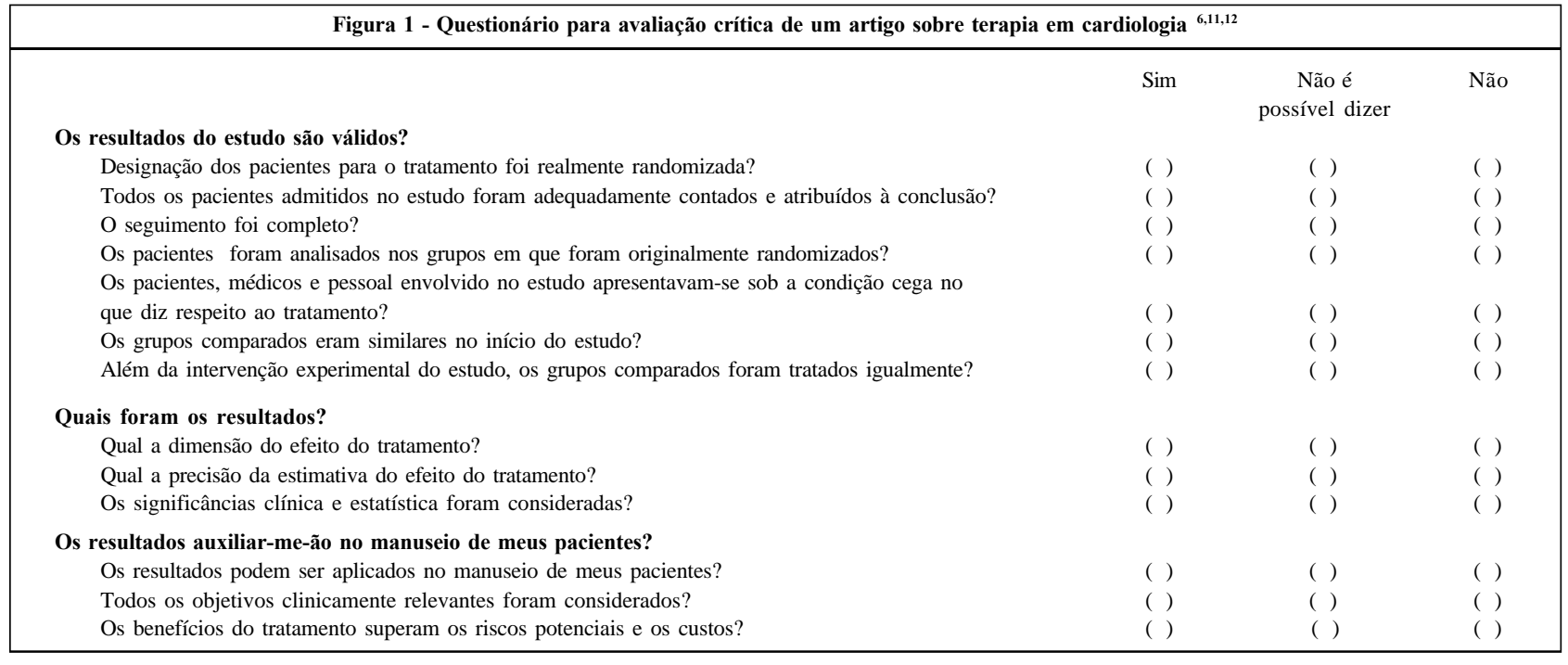

\section{Considerações finais}

Gostaríamos de introduzir o modelo de tomada de decisão clínica a partir do acima exposto no que se refere à terapêutica em cardiologia. Quando concordamos com a validade das informações, quanto a um determinado tratamento, devemos avaliar os seguintes itens antes de incorporá-lo ou recomendá-lo: a) significância estatística ou valor de $\mathrm{p}$ (chance do resultado ter ocorrido por acaso); b) precisão da estimativa de benefício ou intervalo de confiança (quanto mais estreito, mais preciso é o resultado); c) importância clínica ou número necessário para tratar \{NNT (quanto menor, maior a importância clínica) \}; d) cus- to-efetividade (quanto custa para atingir o benefício demonstrado); e) preferências dos pacientes (em determinadas circunstâncias).

A prática da CBE através da aplicação das evidências disponíveis na prática clínica, (epidemiologia clínica à beira do leito) constitui um desafio crescente para todos os cardiologistas e clínicos que praticam cardiologia. Como a globalização do conhecimento médico (informações adequadamente manuseadas gerando conhecimento real) é uma realidade, devemos nos esforçar para homogeneizar nossas condutas, tendo como base um objetivo claramente definido, que é a prática da $\mathrm{CBE}$, ou seja, fazer o correto de maneira correta.

\section{Referências}

1. Fletcher RH, Fletcher SW, Wagner EH - Clinical Epidemiology the essentials. $2^{\text {nd }}$ ed; Baltimore: Williams \& Wilkins 1988.

2. Logan AS - Investigation of Toronto General Practitioner's Treatment of Patients with Hypertension. Toronto: Canadian Facts 1978.

3. Evidence Based Medicine Working Group. Evidence based medicine. JAMA 1992; 368: 2420-5.

4. Sackett DL, Haynes RB, Guyatt GH et al - Clinical Epidemiology-A Basic Science for Clinical Medicine $2^{\text {nd }}$ ed. Boston/Toronto/London: Little, Brown 1991.

5. Sackett DL, Richardson WS, Rosenberg W, Haynes RB - Evidence-based Medicine. How to Practice \& Teach. New York EBM 1997.

6. Avezum A - Cardiologia baseada em evidências e avaliação crítica da literatura cardiológica: princípios de epidemiologia clínica aplicados à Cardiologia. Rev.Soc.Cardiol. Estado de São Paulo 1996; 3: 241-59.

7. Gray JAM - Evidence-based Healthcare. How to make Health Policy and Management Decisions. New York: 1997.

8. Yusuf S, Kitching AD - Evidence-based Cardiovascular Medicine: Why another journal. Evidence-based Cardiovascular Medicine 1997, 1: 1.

9. Haynes RB, Sackett DL, Gray JAM et al - Transfering evidence from research into practice: 2. Getting the evidence straight. Evidence-Based Medicine 1997; 2: 4-6.

10. Yusuf, Kitching AD - From journal to bedside: application of clinical trial results to individual patients. Evidence-Based Cardiovascular Medicine 1997; 1: 29-31.

11. Guyatt GH, Sackett DL, Cook DJ - For the Evidence-Based Medicine Working
Group. User's Guides to the Medical Literature; II. How to Use an Article About Therapy or Prevention - A. Are the results of the study valid ? JAMA 1993; 270: 2598-601.

12. Guyatt GH, Sackett DL, Cook DJ - For the Evidence-Based Medicine Working Group. User's Guides to the Medical Literature; II. How to Use an Article About Therapy or Prevention - B. What were the results and will they help me in caring for my patients? JAMA 1994; 271: 59-63.

13. Altman DG, Gore SM, Gardner MJ, Pocock SJ - Statistical guidelines for contributors to medical journals. In: Gardner MJ, Alman DG, eds - Statistics With Confidence: Confidence Intervals and Statistical Guidelines. London: Br Med J 1989: 83-100.

14. Cook RJ, Sackett DL - The number needed to treat: a clinically useful meassure of treatment effect. Br Med J 1995; 310: 452-54.

15. Yusuf S, Wittes J, Probstfield J, Tyroler HA - Analysis and interpretation of treatment effects in subgroups of patients in randomized clinical trials. JAMA 1991; 266: 93-8.

16. Echt DS, Liebson PR, Mitchell LB et al - Mortality and morbidity in patients receiving encainide, flecainide, or placebo: the Cardiac Arrhythmia Suppression Trial. N Engl J Med 1991; 324: 781-8.

17. The Cardiac Arrhythmia Suppression Trial II Investigators - Effect of the antiarrhythmic agent moricizine on survival after myocardial infarction. N Engl J Med 1992; 327: 227-33. 